Rev. Est. de Políticas Públicas, 6(2): julio - noviembre 2020, 52-64

http://dx.doi.org/10.5354/0719-6296.2020.58621

ISSN edición web: 0719-6296

(C)Copyright 2020: Universidad de Chile, Santiago (Chile)

\title{
Avatares de las políticas públicas en salud mental y su atención sociocomunitaria
}

\author{
Renata Bega Martínez \\ Instituto de Cultura Jurídica (ICJ), Facultad de Ciencias Jurídicas y Sociales (UNLP)
}

\begin{abstract}
Resumen
El presente artículo intentará analizar las políticas públicas destinadas a la salud mental en el territorio argentino, fundamentalmente las relacionadas a la modalidad de atención socio comunitaria y su implementación, una vez establecida ella como uno de los grandes objetivos del Plan Nacional de Salud Mental. En tal sentido, se buscará remarcar sus características y dispositivos principales a lo largo del período 2014-2019 para luego repensar desde una mirada crítica las principales barreras y desafíos en pos de su efectividad como política pública integral, con una mirada orientada en los derechos humanos de los ciudadanos/as a quienes se dirige. Finalmente, se podrá concluir que, pese a las experiencias satisfactorias y los avances significativos en lo propuesto por el Plan Nacional de Salud Mental, la atención comunitaria en salud mental como política pública integral y nacional es hasta el momento un desafío y un objetivo pendiente pero con alternativas posibles para concretarlo.
\end{abstract}

Palabras clave: Salud Mental, Servicios de Salud Comunitaria, Política Pública, Derechos Humanos, Plan Nacional de Salud Mental (Argentina).

Ups and down of public policy in mental health and their social-comunity atention

\begin{abstract}
This article will attempt to analyze public policies aimed to mental health in the argentine territory, fundamentally those related to the socio-community care modality and its implementation once it has been established as one of the main objectives of the National Mental Health Plan; in that matter, it will seek to highlight its main characteristics and devices throughout the 2014-2019 period, and then rethink from a critical perspective the main barriers and challenges in pursuit of its effectiveness as a comprehensive public policy, with a human rights-oriented perspective of the citizens to whom it is addressed. Finally, it can be concluded that, despite the satisfactory experiences and significant progress in what is proposed by the National Mental Health Plan, community care in mental health as a comprehensive and national public policy is up to now a challenge and a pending objective but with possible alternatives to achieve it.
\end{abstract}

Keywords: Mental Health, Community Health Services, Public Policy, Human Rights, National Mental Health Plan.

*Dirección de correspondencia [Correspondence address]:

Renata Bega Martínez, ICJ-UNLP

E-mail: renatabegam@gmail.com

Fecha recepción: 19/09/2020 


\section{Introducción}

La salud mental en Argentina como asunto de salud pública y problemática social ha transitado arduos caminos no solo desde su faceta normativa, representada a finales de siglo XX principalmente con el Código Civil de la Nación y el Decreto Ley 22.914 (Sobre Personas con deficiencias mentales, toxicómanos y alcohólicos crónicos (1983)), sino también desde las políticas sociales propuestas para su abordaje, con nociones y paradigmas adoptados en tal sentido que incidieron inevitablemente en las dinámicas sociales y culturales de la población. Los avances y retrocesos en torno a las políticas de salud mental en ese entonces se vieron fuertemente supeditados al "modelo de Estado Nación y las estrategias de gobierno" (D“Agostino, 2016: 136), en el que, las tensiones público-privado, modelo asilar modelo comunitario y estado de derecho - estado de sitio, generaron notorias inconsistencias en el rumbo elegido y en las políticas sociales que lo llevaron a cabo.

Pese al modelo médico psiquiátrico, hegemónico en ese entonces, los estigmas que atravesaban -y atraviesan- a las personas con padecimientos mentales comenzaron a contar con ciertos avances, primeramente desde la instancia internacional con la Declaración de Caracas (1990) y los Principios para la Protección de los Enfermos Mentales y el Mejoramiento de la Atención de la Salud Mental (1991), lo cual intentó ser acompañado en distintas provincias de la República de modo aislado. En tal sentido, la experiencia emblemática impulsada por la Provincia de Rio Negro desde 1985 en lo referido a modelos de atención de la salud mental en ámbitos socio-comunitarios, el trabajo desde la interdisciplina y el foco en la integración de las personas con padecimiento mental, tomó cierto reconocimiento internacional y logró el 11 de septiembre de 1991 la sanción por la Legislatura Provincial de Rio Negro de la ley 2440 de "Promoción Sanitaria y Social de las Personas que Padecen Sufrimiento Mental", pionera a nivel local de lo que años después llegaría a nivel nacional en torno a los diversos dispositivos de atención, el paradigma biopsicosocial y por sobre todo la mirada puesta en los derechos humanos fundamentales de todas las personas.

Iniciado el siglo XXI los cambios comenzaron a ser mayores; desde el ámbito normativo, las declaraciones internacionales y el profundo trabajo de Naciones Unidas para erradicar la discriminación repercutieron en el ámbito nacional. Fue el año 2008 con la ratificación de la Convención sobre los Derechos de Personas con Discapacidad (CDPD -2006) el momento clave que estableció formalmente una nueva mirada acerca de la discapacidad mental e intelectual y los padecimientos mentales en el país.

Consecuentemente, en el año 2010 se sanciona la Ley Nacional 26.657 de "Derecho a la Protección de la Salud Mental" (usualmente mencionada como Ley Nacional de Salud Mental, de aquí en más LNSM) con un notorio enfoque en los derechos humanos de las personas y en el concepto social de discapacidad, lo cual llevaría a modificar no solo el plexo normativo nacional y provincial, sino los paradigmas predominantes $^{1}$, la atención en salud mental y las políticas públicas en general que abordan la temática. La norma sin duda vino a legitimar una práctica que durante años se sostuvo de manera aislada desde espacios de atención e instituciones de diversas localidades de la Argentina, con el sustento en leyes provinciales y con la intención de los espacios socio-sanitarios de comenzar a reformar sus prácticas, pese a no contar con la normativa nacional que lo regule y avale.

Establecida la salud mental como asunto de salud pública, sus políticas y modelos de atención se supeditan a los sistemas de atención médica dentro de los sectores estatales, privados y las prestaciones de obras sociales, que limitan y a la vez complejizan su instrumentación.

Las nociones que se abordarán en este artículo pretenden contar con un enfoque en lo ut supra mencionado y con una clara perspectiva de derechos humanos. Es por lo cual, el concepto de salud mental tendrá en cuenta los avances normativos y los paradigmas vigentes y se entenderá como el proceso determinado por componentes históricos, socio-económicos, culturales, biológicos y psicológicos, cuya preservación y mejoramiento implica una dinámica de construcción social vinculada a la concreción de los derechos humanos y sociales de toda persona $^{2}$.

En tal sentido, al mencionar la modalidad de atención socio-comunitaria debe focalizarse en los cui-

\footnotetext{
${ }^{1}$ Entendidos como los saberes hegemónicos en ese entonces de disciplinas tales como psicología, psiquiatría, derecho, trabajo social, entre otros.

${ }^{2}$ Articulo 3 Ley Nacional de Salud Mental y Adicciones $\mathrm{N}^{\circ}$ 26657. En línea con los postulados de la Organización Mundial de la Salud, la CDPD y su respectivo Comité, las redes de usuarios y usuarias y las referencias bibliográficas del presente artículo.
} 
dados y abordajes con énfasis en el territorio, en la población de la cual forma parte la persona que requiere de tales servicios, entendiendo que ello se encuentra íntimamente ligado al concepto social de discapacidad y al enfoque de derechos humanos que se encuentra en los instrumentos analizados, enfoque que se tomará como marco conceptual y desde una mirada en el derecho internacional de los derechos humanos, tal como lo abordan doctrinarios/as mencionados/as en este trabajo (Abramovich y Pautassi, 2009).

El objetivo central propuesto aspira a indagar acerca de las políticas públicas diseñadas y llevadas a cabo en el período 2014- 2019 en lo que refiere a la atención en salud mental comunitaria en Argentina, para las personas que actualmente se encuentran en un hospital neuropsiquiátrico, así como para las personas que requieren de los servicios de salud mental en su comunidad. Éste objetivo se rodeará de objetivos específicos tales como el exhumar el Plan Nacional de Salud Mental (PNSM) y su factibilidad, analizar si el PNSM es compatible con el ordenamiento jurídico nacional e internacional mencionado y examinar si es acaso posible llevar a la práctica en el territorio lo indicado por el PNSM.

Para ello se utilizarán como fuentes primarias de análisis los textos de la LNSM, la CDPD y el PNSM y como fuentes secundarias informes y guías de organizaciones y agrupaciones civiles referentes en la temática, así como informes y complicaciones de las áreas de gobierno a cargo del PNSM en el período indicado, y estudios de doctrina esenciales en áreas tanto de políticas públicas como de salud mental y derechos humanos.

El trabajo presenta una primera instancia de aproximación a las fuentes primarias y a la noción de políticas públicas que se tomará a lo largo de estas páginas, para luego comprender las particularidades de los servicios de salud mental en la Argentina y los avances -así como las principales dificultades- en lo que refiere a los servicios destinados a la salud mental comunitaria y la territorialización de los mismos. En esta segunda instancia, la mirada estará puesta en los derechos humanos fundamentales de los y las usuarias de los servicios de salud, sus posibilidades, la interdisciplina que requiere el abordaje comunitario, los recursos que se disponen y los vínculos afectivos.

Ello derivará en comprender que el camino recorrido y las propuestas del PNSM resultan acordes al enfoque de derechos humanos y de salud mental comunitaria que se propone en los instrumentos normativos, pero se comprenderá también que no pueden entenderse que tales enfoques y los objetivos del Plan estén enteramente cumplidos. Puede darse cuenta de ciertas experiencias marco así como recursos que pueden ser considerablemente significativos para avanzar, pero resulta preciso continuar para generar políticas públicas integrales de aquí en adelante.

\section{La norma y la legitimación de los "nuevos" dispositivos}

Respecto a los padecimientos mentales y sus modalidades de abordaje, fue desde el ámbito internacional donde el cambio ha sido significativo y ha impulsado la réplica en los ordenamientos jurídicos nacionales. Junto a todo lo que la CDPD representa en cuanto a la mirada puesta en la necesaria protección, reconocimiento y promoción de los derechos humanos de las personas con discapacidad, tal instrumento convencional en su artículo 19 reconoce explícitamente el derecho a una vida independiente y la inclusión en la comunidad de toda persona pese a las circunstancias en las que se encuentre, con la obligación de adoptar desde el Estado las medidas efectivas y pertinentes que facilite el pleno goce de derechos humanos y la potestad de elegir el sistema de vida que desean llevar, así como el acceso apropiado a múltiples servicios de asistencia.

La CDPD ha sido incorporada en el ordenamiento jurídico argentino y con jerarquía constitucional, de todas maneras, fue la LNSM el hito que definió las nuevas formas de abordaje y la nueva mirada en salud mental como política pública.

En el presente trabajo se pretende investigar si efectivamente el contenido de la normativa, tanto nacional como internacional, se vio y se ve aplicado como política pública en materia de salud mental, sobre todo en materia de salud mental comunitaria. Para ello, se hará especial énfasis en las personas institucionalizadas en hospitales neuropsiquiátricos que deben reconfigurar su modalidad de atención para adecuarse a los nuevos lineamientos adoptados. También en lo que ocurre en las personas con padecimiento mental, que, pese a no estar institucionalizadas, deben recibir algún tipo de atención en urgencias así como en tratamientos prolongados y en principio no debería ello ocurrir en hospitales monovalentes. 
Establecida la posición de la CDPD en la materia, el contenido de la LNSM siguió su rumbo y se convirtió en el instrumento normativo nacional específico en salud mental y sus abordajes.

La Ley en su articulado refuerza lo establecido por la CDPD y otros instrumentos internacionales y añade particularidades que hacen al quid de la cuestión reflejando la importancia de la interdisciplina, la atención en un ámbito que no implique la internación en hospitales generales -siendo este el último recurso (art. 14 y 28 LNSM)-, la base en la atención primaria en salud y en la salud mental comunitaria. Remarca la prohibición de crear nuevos manicomios -así como que los existentes deberán adaptarse a los nuevos objetivos y principios-, su sustitución por los nuevos dispositivos, la mínima medicalización posible y la inclusión de la persona en su ámbito socio-cultural, económico y afectivo, entre otros. Ello acompañado por su decreto reglamentario 603/2013 significaron -y significan- una nueva mirada en salud mental en el territorio argentino.

Ahora bien, con la vista puesta en las modalidades y dispositivos de atención que el Estado debe garantizar una vez sancionada la LNSM, pese a tener en claro el universo normativo resulta trascendental analizar cómo llevar tal iniciativa a la práctica. La noción de bienestar público implica un esfuerzo en las distintas esferas gubernamentales para asegurar el efectivo goce de derechos de toda la población; el contenido de la Ley mencionada tuvo tal intención, pero para llevarla a hechos concretos de la vida de las personas se requiere "una acción pública que se realiza con recursos que también son públicos" (Vázquez y Delaplace, 2011:36). Estas acciones gubernamentales y colectivas concernientes a diversos aspectos de interés público pueden ser consideradas como políticas públicas, que pretenden diseñar, aplicar y evaluar de forma justificada y racional tales medidas -con el bienestar social como norte-.

Una vez que se asume a las políticas públicas "como parte de las obligaciones estatales para el cumplimiento efectivo de los derechos humanos" (Abramovich y Pautassi, 2009:1) que excede la mirada benéfica y asistencial e incorpora las ideas de institucionalidad y gobernabilidad, resta considerarlas también como ciclos que se realimentan constante y sistemáticamente tal como lo mencionan Vázquez y Delaplace (2011), que surgen desde que se nota una necesidad y se incorpora en la agenda pública ${ }^{3}$; las problemáticas que atraviesan a la salud mental comenzaron a incorporarse una vez establecidas en el ámbito normativo, acompañadas de la fuerza de organizaciones sociales y no gubernamentales para visibilizarlo, hasta llegar exitosamente a oídos de las esferas burocráticas. Uno de los problemas consistía en las ya mencionadas modalidades de atención en salud mental y lo que el Estado desde sus hospitales monovalentes ofrecía para la vida de los anteriormente denominados "pacientes"; con el artículo 11 de la LNSM el deber ser luego del 2010 se modifica y deben de proveerse:

Dispositivos tales como consultas ambulatorias; servicios de inclusión social y laboral para personas después del alta institucional; atención domiciliaria supervisada y apoyo a las personas y grupos familiares y comunitarios; servicios para la promoción y prevención en salud mental, así como otras prestaciones tales como casas de convivencia, hospitales de día, cooperativas de trabajo, centros de capacitación socio-laboral, emprendimientos sociales, hogares y familias sustitutas.

Las alternativas señaladas por el articulo $n^{\circ} 11$ son algunas de las soluciones posibles al problema de la internación cronificada y los dispositivos de atención vetustos y violatorios a derechos humanos fundamentales que se ofrecían previo a la LNSM. Para acompañar el diseño de estos dispositivos se precisó de políticas públicas, pese a que en tal intervención se evidencien matices político-partidarias, ideológicas y profesionales; reconociendo que cada política pública se encuentra situada en una política gubernamental. Efectivamente, "la salud es política porque sus propios determinantes sociales son sensibles a las intervenciones políticas" (Franco-Giraldo y Âlvarez Dardet, 2008:282), pero, tal intervención será positiva en gran parte si se basa en los derechos humanos.

Para reforzar las acciones estatales, Naciones Unidas ha recomendado a los Estados la elaboración de planes de acción nacionales que busquen una mejora y una protección en materia de derechos humanos. En tal sentido, en Argentina una vez establecido el ámbito normativo y las instituciones principales que habrán de aplicarlo (Ministerio de Salud y Dirección Nacional de Salud Mental y Adicciones)

${ }^{3}$ En Dirección Nacional de Salud Mental y Adicciones (2018a) se recuerda que el $22 \%$ de la carga de enfermedad en América Latina y el Caribe incluye padecimientos mentales y que tal cifra va en aumento. 
se decidió reforzar las estrategias nombradas en la LNSM con la gestación del Plan Nacional de Salud Mental (PNSM) que durará desde su fecha de puesta en marcha en octubre del año 2013 hasta el 31 de diciembre del $2020^{4}$.

El PNSM establece los principales problemas observados en la materia así como los objetivos principales que se perseguirán en cada uno de ellos para resolverlos. Junto a la estigmatización, los consumos problemáticos, las infancias y juventudes, el monitoreo e investigación y la formación en recursos humanos, se identifica un problema fundamental en el PNSM que hace a la temática abordada por este trabajo y es que: "La red de Salud/Salud Mental es inadecuada, cuando no inexistente, a la modalidad de abordaje que plantea la Ley Nacional de Salud Mental № 26.657 y su Decreto Reglamentario № 603/2013" (Dirección Nacional de Salud Mental y Adicciones, 2013: 22).

Para resolverlo plantean como objetivos: el fortalecimiento de la red de salud mental que adecue sus servicios y dispositivos a la nueva Ley, la apertura programada y gradual de servicios en hospitales polivalentes, el cierre de las instituciones totales (colonias, hospitales, comunidades terapéuticas, clínicas psiquiátricas, entre otros), el fortalecimiento de la atención primaria en salud, le redistribución de recursos humanos y financieros hacia los nuevos dispositivos, la circulación de información acerca de las nuevas ofertas del sistema de salud, entre otros.

Con las pautas generales del PNSM para reformular las practicas institucionales, en el ámbito de salud pública y hacia una base comunitaria, se adoptó como meta a largo plazo que el $100 \%$ de las personas con padecimiento mental que se encuentren institucionalizadas con más de 2 años en efectores públicos sean externadas e incluidas en la comunidad para noviembre del año 2020. Del mismo modo, que el $100 \%$ de los dispositivos comunitarios creados a partir de la puesta en vigencia del presente Plan que integran la red de servicios de Salud Mental en las distintas jurisdicciones, se encuentren comprendidos en los principios de la LNSM.

\footnotetext{
${ }^{4}$ Resulta importante rescatar que el PNSM fue elevado en octubre de 2011 a las autoridades del Ministerio de Salud de la Nación, luego de un proceso de elaboración colectiva y de la lucha de organizaciones no gubernamentales y asociaciones civiles; sin embargo, fue aprobado y promulgado recién en mayo de 2013, debido a los diversos prejuicios y barreras de las corporaciones médicas hacia el Plan y lo que en su instrumentación se proponía.
}

A partir de la implementación de las estrategias reflejadas en políticas públicas comenzará el cuestionamiento acerca de si son ellas adecuadas y, sobre todo, efectivas en la eliminación de las "características custodiales del modelo de atención" (De Lellis, 2013: 10) con el fin de lograr una mayor cercanía a la comunidad desde los servicios adecuados para transformar lazos comunitarios en "vínculos solidarios hacia la constitución de la propia comunidad como sujeto activo de transformación de sus realidades, generando condiciones comunitarias propicias para la toma de decisiones autónoma y conjunta sobre el propio proceso de salud-enfermedad-cuidados" (Orellana, 2019) Principalmente con el protagonismo de la atención primaria entendida como "la forma más integral, positiva y democrática de construir el cuidado de la salud y favorecer la equidad. Los procesos de territorialización contribuyen a facilitar la accesibilidad a los cuidados requeridos" (Gerlero, Augsburger, y Duarte, 2011: 24).

Junto al PNSM, el Comité sobre los Derechos de las Personas con Discapacidad de Naciones Unidas (CDPD-ONU) manifestó en 2012 su "preocupación por la falta de recursos y servicios adecuados y suficientes para garantizar el derecho de las personas con discapacidad a vivir de modo independiente y ser incluidas en la comunidad" (Asociación Civil por la Igualdad y la Justicia, 2018:4) por lo que, instó a que en Argentina se desarrollen todos los programas y ofertas necesarias para cumplir con sus compromisos y con el articulado de la LNSM (particularmente su art. 11).

\section{Del PNSM a las políticas en terri- torio}

Frente a todo lo establecido podría decirse que en el periodo analizado se ha delineado una política de salud mental, entendida como el "conjunto organizado de valores, principios y objetivos para mejorar la salud mental y reducir la carga de trastornos mentales de la población" (Organización Mundial de la Salud, 2005:12) tanto para la actualidad como para un futuro mediato, definiendo las prioridades de cada gobierno y entendiendo que las decisiones tomadas "son acciones que afectan y limitan la capacidad de decisión sobre las acciones que se tomarán mañana" (Del Valle y Boga, 2016:6). En el plano de lo ideal, se espera que las prácticas en salud logren tal integralidad y organización que no sea siquiera preciso aclarar a qué modelo sanitario responde. $\mathrm{Pa}-$ 
ra ello, las políticas públicas deben cumplir con la norma y dar "racionalidad al uso de recursos escasos en el cumplimiento de las actividades estatales en cada una de las partes que integran el ciclo de vida" (Vázquez y Delaplace, 2011: 40) como su objetivo último. Sin embargo, pese a que resulte un enunciado razonable, ello nada establece sobre cuáles son las acciones y políticas correctas, lo que en cierto punto queda ajustado al libre albedrio en la elección y posterior evaluación de su eficiencia y eficacia, con el riesgo de convertirse en indicadores vacíos de contenido.

Las iniciativas impulsadas en el marco del PNSM pese a que "el sistema de salud argentino posee como característica estar fuertemente fragmentado y desarticulado debido a que operan tres subsectores de atención a la salud (Público, Obras Sociales y Privado)" (Sola, Martínez Calle, y Cuello, 2015:20) pueden ser simplificadas en pos de su unificación y de pensar, en un primer esquema, en el remplazo de la atención en hospitales monovalentes a hospitales polivalentes y en los centros de día, las casas de medio camino, entre otros espacios en la comunidad, con un mayor ingreso en los territorios. Abordando la salud con mayor énfasis en los vínculos, en la comunidad y en la continuidad.

Cabe remarcar que a estos dispositivos acuden personas que están en proceso de externación -lo cual cuenta con ciertas dificultades particulares- y personas que acuden al sistema de salud por un episodio agudo/crisis donde el equipo de salud busca encontrar alternativas en el tratamiento acorde a la normativa vigente. Las políticas públicas en salud mental tuvieron como objetivo que la circulación de usuarios/as en los niveles del sistema de salud sea fluida y significativa en el cambio del paradigma asilar- tutelar hacia el paradigma comunitariopromotor de derechos humanos; pese a no contener reglamentos y protocolos uniformes, tomando en cuenta que la LNSM es de orden público, las realidades regionales no opacan el deber nacional de que existan hospitales polivalentes y espacios en la comunidad.

Entonces, uno de los ejes estará puesto en la atención en los hospitales generales; tal como lo establece la Dirección Nacional de Salud Mental y Adicciones: "se encuentra ampliamente probado que el abordaje de la salud mental dentro del ámbito de atención en un hospital general disminuye el riesgo de segregación, cronificación, abandono y de abusos a la integridad psicofísica" (2018a: 11) de los/as usuarios/as, con un foco en la integralidad, la interconsulta, la no permanencia y una mayor adherencia a los tratamientos con una óptima resolución de las urgencias. En tal sentido, los hospitales públicos deben contar con cierta estructura para que ello ocurra, debe existir un espacio e infraestructura adecuada para efectivizar tal tratamiento -lejos de las estructuras que conforman un hospital monovalente--, con acceso al resto de las áreas, pero respetando las medidas y las normas de seguridad y bio-seguridad, en respeto de las personas en el área de salud mental y del resto de las personas que se atienden en el hospital, disponibilidad de los/as trabajadores/as para una atención que se adecue a las necesidades de quien padece, dispositivos administrativos y tecnológicos aptos para tal modalidad de atención ${ }^{5}$, cuerpo de profesionales y operadores/as completo que incluya trabajador/a social, medico/a, psicólogo/a, enfermera/o, acompanante y asistente terapéutico/a, entre otros.

En estos hospitales se encuentra un nuevo desafío, quizás el más significativo, que implica salir de la lógica adoptada durante muchas décadas y pensar en un espacio hospitalario que exceda a la institucionalidad, que busque nuevas alternativas y que atienda las demandas espontáneas desde un lugar de mayor empatía, integralidad y en respeto a los derechos humanos de quienes acuden.

Por otro lado, existen otras tantas alternativas sociocomunitarias dentro de las políticas públicas en salud que enfocan a la continuidad del tratamiento en centros $u$ hospitales de día y de noche, con dispositivos para la inclusión socio-laboral, habitacional, artísticos, entre otros. En esos casos, los dispositivos se encuentran cercanos a donde habita la persona y en su ámbito de comunidad, suelen conformarse grupos y participar en talleres que impliquen una (re)inclusión a los hábitos sociales que a su vez refuercen las posibilidades laborales y la autonomía ${ }^{6}$, abriendo, como se expresa en Orellana "aristas que en la internación fueron vetadas, tales como la participación comunitaria y política, la convivencia,

\footnotetext{
${ }^{5}$ Ello incluye agilizar la recepción y admisión de usuarios/as, la atención interdisciplinaria las 24 horas, la internación, la atención de la demanda espontánea, la ambulatoria programada, la psicoterapia, el tratamiento psicofarmacológico, la interconsulta, el hospital de día, la evaluación del diagnóstico, el plan terapéutico, la reunión de equipos, la continuidad y cuidados para el seguimiento, acciones de promoción y prevención en salud, entre otras, con todo lo que esto implica.

${ }^{6}$ Las experiencias suelen ser talleres literarios, huerta, carpintería, cocina, teatro, entre tantos otros, con la participación de acompañamiento terapéutico y de organizaciones civiles para hacer posible el espacio y mantenerlo.
} 
el autovalimiento y como horizonte el autoreconocimiento como sujetos de derecho" (Orellana, 2019: 92). Todo ello requiere un anclaje profundo al territorio y a los ámbitos municipales, así como la construcción de espacios en condiciones adecuadas, las consultas pertinentes y los/as trabajadores/as disponibles, la calidad de atención y la reducción de la espera, las mismas tareas de control de medicación, admisiones, apoyo, acompañamiento en el proceso judicial-de existirlo-, desplazando barreras como el horario reducido de estos centros, no contar muchas veces con presupuesto propio y relacionarse de manera estrecha con los monovalentes al compartir recursos y personal, entre otras.

Más allá de que este trabajo no profundice en el derecho a la vivienda adecuada y en las pensiones ofrecidas por el Estado a personas en situación de vulnerabilidad, resulta preciso remarcar que la no satisfacción de derechos económicos, sociales y culturales para personas con padecimientos mentales y/o discapacidad mental e intelectual "representa una deuda de carácter histórico que va progresivamente en aumento" (Dirección Nacional de Salud Mental y Adicciones, 2018b: 13). Muchas veces son estas carencias las que perpetúan la permanencia de los hospitales neuropsiquiátricos o el regreso al manicomio de las personas desinstitucionalizadas.

Las situaciones de vulnerabilidad también son subsanadas con políticas públicas, en muchos casos son estas carencias las que generan el padecer, si se toma el concepto social de la salud mental. Las desventajas y la negación en derechos básicos son malestares que atraviesan a la persona de manera transversal y, aunque no sea el eje del trabajo actual, deben señalarse como un gran ítem que choca con los tratamientos en salud desde la comunidad y es un potencial obstructor.

Desde REDI (2011) se ha hecho especial hincapié en la búsqueda de la autonomía de las personas con discapacidad mental e intelectual; pese a los avances aun los nuevos dispositivos adoptan una mirada paternalista y medicalizante, pero la lucha debe continuar y perseguir el fin inmediato de la calidad en los servicios ofrecidos que se conviertan en herramientas para saltar barreras, no para profundizarlas. Es por ello que deben acompañarse tales medidas con "programas, proyectos y acciones de prevención y de promoción de estilos de vida saludable con el fin de evitar que los problemas de salud mental aparezcan" (Cohen, 2015:14).
En ese sentido, el fin último es generar una Red en salud mental consolidada, que exceda el asistencialismo y potencie a la persona, encontrándose probado que esta forma es la respuesta ética y científica adecuada para combatir los padecimientos subjetivos. Garantizando la disponibilidad, accesibilidad, calidad y poder de adaptación para cada usuario/a, con políticas integrales que abarquen la perspectiva de género, las infancias y juventudes, adultos/as mayores, situaciones de pobreza extrema y abandono. Para así ubicar a las personas en un ámbito donde se sientan cómodas y se parta desde la igualdad y la no discriminación.

\section{Tensiones y deudas en su puesta en marcha}

Como lo expresa Morasso "el $75 \%$ de los países latinoamericanos tienen plan de salud mental; sin embargo los índices de implementación son bajos: más de un $50 \%$ de los países tienen valores de implementación menor al 50\%" (2018): 18); las causas de este déficit podrían ser múltiples y es aquí donde intentarán señalarse algunas.

En muchos casos los dispositivos alternativos al hospital monovalente (hospitales generales y espacios sociocomunitarios como se han mencionado) suelen encontrarse con barreras a la hora de implementar lo que establece el PNSM y lo que se consideran como ejes en las políticas públicas. La LNSM fue sancionada en un contexto político e ideológico muy distinto al momento de que fue efectivamente implementada en la práctica, los ejes centrales del gobierno de turno cambiaron y ello implico una diferencia en el destino de los recursos humanos y, sobre todo, económicos.

La modificación en la estructura del Ministerio de Salud y de la Dirección Nacional de Salud Mental junto con el cambiante entorno socioeconómico y la dificultosa participación de organizaciones no gubernamentales terminaron por reducir la fuerza del impulso del proyecto. La población con padecimientos mentales no decrecía y era allí donde se esperaba que en casos de crisis se provea una atención en hospitales polivalentes y en casos de menor complejidad la atención en niveles primarios en salud, desafortunadamente éstas no se han consolidado como únicas respuestas ni como adecuada contención y tratamiento y en las urgencias se "impulsaban a los profesionales que se desempeñaban en el ámbito 
de los hospitales generales a efectuar la derivación a instituciones monovalentes" (De Lellis, 2013:11).

Es la escasez de recursos económicos lo que en principio impide una mejora en la estructura de salud comunitaria ${ }^{7}$ que, por su descentralización dentro de cada provincia y por las diferencias en cada región "con inequidades en el acceso como corolario" (Gerlero et al., 2011: 25), se termina por dirigir los recursos existentes al mantenimiento de las todavía vigentes instituciones monovalentes, las cuales deberían ser sustituidas y designar esos ingresos -económicos y humanos- a dispositivos de la Red Comunitaria.

Los altos costos de los psicofármacos, la falta de pensiones y obras sociales que cubran de manera integral las necesidades de la persona padeciente, la distancia geográfica del usuario/a con los dispositivos y la fácil ocupación de los cupos dentro de cada centro de salud, el sueldo por debajo de lo esperable en los equipos tratantes así como la falta de disponibilidad horaria, la imposibilidad de ampliar los espacios participativos y el dialogo colaborativo, son algunos de los ítems que atrasan la integralidad de las políticas públicas (Cohen, 2015; Gerlero et al., 2011; Morasso, 2018). Paradójicamente, son las políticas públicas y económicas las que pueden solucionar tales falencias, en ese sentido "se podría afirmar que el problema de la representación de intereses, en realidad no se resuelve, sino que se financia" (Del Valle y Boga, 2016:9).

Ello se enfatiza en las mujeres que intentan acudir al modelo de salud comunitario donde las problemáticas se duplican al encontrarse con menores posibilidades de salir del espacio doméstico, de solicitar el "cuidado" del Estado cuando es ella quien debe asumir el rol de cuidadora, de tener pensiones que sean ajenas a su calidad de madre, de conseguir un trabajo al no solo ser mujer sino que ahora es una "mujer -loca", de poner en riesgo su capacidad para continuar con la crianza de sus hijos/as, entre otras; allí no se encuentran dispositivos integrales.

En líneas generales, pese a contar con el PNSM, no se presentan aun políticas públicas consolidadas; los protocolos y reglamentaciones suelen surgir de espacios autoconvocados, del mismo modo surge la incorporación de otras disciplinas que el Estado hasta ese entonces no podía costear. La accesibilidad y

\footnotetext{
${ }^{7} \mathrm{El}$ primer paso sería garantizar personal de trabajo de diferentes disciplinas y roles, psicofármacos, insumos, infraestructura adecuada, etc.
}

la calidad hacen al deseo y a la continuidad del/la padeciente en su proceso de recuperación; por tal motivo y en vistas de la continuidad y la convivencia de espacios manicomiales con espacios comunitarios resultan pertinentes los interrogantes "¿La lógica manicomial existe solo en las instituciones psiquiátricas? ¿Cómo derribar estos muros? ¿Cómo esfumar fronteras imaginarias causantes de ruptura de lazos? y ¿Cuál es el nivel de influencia y determinismo de estos mecanismos, en las problemáticas que escuchamos cotidianamente y que se enquistan bajo el rótulo de "las enfermedades"?" debidamente planteados por Aquila et.al (2015: 123).

\section{Factores y determinantes sociales indispensables}

La formación y capacitación continua en salud mental de los y las trabajadoras, agentes y profesionales de la salud mental implica uno de los problemas centrales dentro del PNSM. Aunque ello no sea el eje central del trabajo, debe considerarse, dado que de esa formación surgirán trabajadores/as con perspectiva de derechos humanos y a tono con los postulados de la LNSM y su Decreto Reglamentario (Dirección Nacional de Salud Mental y Adicciones, 2013). Ello refiere necesariamente a una política pública, que garantizaría la calidad en la atención en salud.

Para tal tarea, resulta imprescindible readecuar el currículo universitario y de demás instituciones formadoras en salud así como capacitaciones continuas en todos los equipos interdisciplinarios que abarquen todas las aristas que afectan a la salud mental de las personas. La formación implica también en la práctica cotidiana promover espacios de escucha atenta, de articulación entre equipos, de intersectorialidad, estimulado desde "lo público" y capacitaciones con el punto en descubrir qué es lo que resta por hacer y cuáles son las estrategias a plantear frente a los equipos en salud y lo que ellos tienen para aportar al estar inmersos en la dinámica del día a día.

"Las trabas institucionales, la sobrecarga de trabajo y el desgaste natural de los equipos contribuyeron a que muchas actividades se discontinuaran... dicha medida de por sí, no garantiza la ruptura y superación de prácticas autoritarias, verticalistas, individualistas y descontextualizadas de las situaciones económicas, sociales y cultura- 
les de las personas con padecimiento mental y sus familias" (Cohen, 2015: 14).

Para una mejora dentro de esta coyuntura se requiere del apoyo y el accionar de autoridades de gobierno, organizaciones gremiales y poderes corporativos que fomenten la formación, los derechos de las personas trabajadoras y las investigaciones en la temática; pese a que todas las modalidades de atención deberían seguir una línea común y lo que varíe sea únicamente la densidad y la estructura de acompañamiento de la Red en casos complejos, de todas maneras, previo a los dispositivos mencionados, la primer alternativa deberían ser los centros de salud del primer nivel de atención y sus equipos interdisciplinarios sin siquiera tener que acudir a un hospital general o una casa de medio camino.

Otro factor para repensar es la justicia -entendida aquí principalmente como fueros de primera instancia en materia civil y comercial y de familia, junto con la curaduría oficial - y cómo ella atraviesa a las personas en la determinación de sus capacidades y el modelo de vida que deciden llevar, pese a que en la normativa actual sea potestad del equipo de salud y de la persona involucrada el sistema de vida y el tratamiento que consideran adecuado. Asimismo, la intervención del fuero penal -con sus particularidadescuando se interviene en la declaración de inimputabilidad de una persona que ha cometido un delito, debiendo ser tal situación revisada a la luz de los parámetros y principios de la normativa vigente, incluyendo la LNSM. Está claro que las dificultades que atraviesan al poder judicial no son las mismas que las que atraviesa el sistema y los equipos de salud; muchas veces las decisiones de uno influyen en las del otro para alivianar o densificar el proceso, en eso existen experiencias de ambas matices y en muchas ocasiones el articular de estos actores que indefectiblemente participan en el proceso ha derivado en consensos, en optimización de tiempo y de recursos, en diálogo y en experiencias positivas con un tratamiento sostenido en bases comunitarias pero muchas otras veces los distintos lenguajes, falta de tiempos e imposibilidades de garantizar las cuestiones básicas ${ }^{8}$ impidieron buscar modos alternativos y las personas continuaron en el encierro manicomial o en lógicas similares.

\footnotetext{
${ }^{8}$ En muchos casos, la persona no cuenta siquiera con Documento Nacional de Identidad, no tienen certificado único de discapacidad, pensión no contributiva, obra social, etc.
}

De considerar una propuesta articulada con estos dos puntos centrales no debe perderse de vista la óptima participación de espacios ministeriales como autoridades de aplicación y de poderes legislativos, con un sostenido discurso y propuesta aferrada al paradigma de salud comunitaria; ello se liga necesariamente a las políticas públicas y, aunque parezca lo más seguro y de difícil regresividad, es necesario recordar que en el año 2017 un proyecto de reforma del decreto reglamentario 603/13 amenazó en retroceder sobre los derechos de las personas con padecimiento mental intentando reinstalar las antiguas modalidades de atención y conceptos biologicistas que influirían en la noción y realidad de los/as usuarios/as. Es por situaciones como tales que debe de considerarse este factor como central para mantener un equilibrio, una sustentabilidad y una sostenibilidad en las políticas públicas.

No está de más decir que ésta y todas las políticas deben basarse en los principios de universalidad del acceso, integralidad de la respuesta y equidad en salud sin distinciones arbitrarias que vulneren los derechos humanos y pongan en clara desventaja a personas que se encuentran en situación de vulnerabilidad; toda persona tiene derecho a recibir prestaciones esenciales que garanticen el derecho a su salud, ello se encuentra establecido no solo en la Constitución Nacional sino por sobre todo en los tratados internacionales que cuentan con jerarquía constitucional -así lo asevera, por ejemplo, la mencionada CDPD-.

Aunque la LNSM y la CDPD acompañen los principios de universalidad, integralidad, equidad y accesibilidad, es preciso remarcar que tales avances en los principios no solo deben verse plasmados en la norma sino también en las políticas públicas. Claros los dispositivos expuestos en el trabajo y su implementación, hay que ligarlos con otros aspectos que acompañan el tratamiento y lo constituyen pese a no ser estrategias en salud per se -si se piensa en la salud como un proceso social y no solo biológico-.

Las personas que pretenden ser desintstitucionalizadas o las que deben recuperarse de crisis agudas y han acudido a dispositivos comunitarios deben ser acompañadas con la posibilidad de mejorar sus condiciones de trabajo, de formación y de escolaridad; como expresa Cea Madrid existe una carencia "en términos de acceso a oportunidades de educación y empleo, así como escasos apoyos para promover la participación en la vida política y el ejercicio de la libertad de asociación" (Cea Madrid, 2019:3). La in- 
clusión social debe pensarse mediante el derecho al trabajo y el acceso a una actividad productiva con justa remuneración, para ello debe acompañar el Estado en el desarrollo de "competencias laborales que respondan a demandas de trabajo actuales" (Vitelli y Fruttero, 2018: 118) -no ponerlo como una carga o responsabilidad de estas personas, ni que sea un problema individual, que se encuentran siempre con barreras al querer tomarlo como un desafío propioya que el trabajo es una práctica que "crea subjetividades, reconstruye lazos y construye nuevos, dignifica a la persona, proporciona recursos económicos para su sostén, promueve autonomía y rompe con estigmatización y discriminación" (Vitelli y Fruttero, 2018: 119).

Frente a tales necesidades en épocas neoliberales "no debe ser ni el mercado ni la sociedad civil quienes tienen la responsabilidad de tomar la iniciativa o decisiones de fondo en estas áreas" (Levy, 2007: 3), el Estado conoce las posibilidades de cada habitante de su territorio y atiende las coyunturas y las carencias que ha atravesado y atraviesa cada persona debiendo -probablemente a futuro y como arista que acompaña el objetivo principal -resignificar "el trabajo", sus condiciones y sus fronteras; pensando aquí en un genuino cooperativismo y autogestión (Levy y Bermúdez, 2012) como interesante salida del laberinto al menos para los sujetos/as que se relacionan a las temáticas y situaciones planteadas en este trabajo.

Lo cierto es que, en los procesos mencionados a lo largo de estas páginas, el rol que ocupan los vínculos afectivos es igual de fundamental que la educación y el trabajo para garantizar el derecho a la salud; en muchos casos no se toma en cuenta su verdadera importancia e influencia a la hora de pensar un tratamiento en la comunidad y por sobre todo con sus afectos presentes. Apelando a la contundencia de las palabras de Battaglia et. al "las redes sociofamiliares quedan ubicadas en situaciones de "todo o nada" respecto del cuidado de las personas dependientes o semidependientes" (2019: 33) en coyunturas como las descriptas; la crisis del cuidado está planteada frente a múltiples escenarios y éste es uno de ellos.

En muchos casos frente a la pregunta "¿por qué las familias no se los llevan?" (Battaglia et al., 2019), aplicable tanto al egreso de una persona de una institución total o de un centro de atención con base en la comunidad, es necesario comprender que el apoyo de las familias muchas veces se supedita al factor económico como el factor central. Muchas veces por falta de dinero o por deber sostener horarios laborales resulta imposible generar un vínculo -o recomponerlocon la persona que está en un tratamiento complejo, de la misma manera que suele esperarse que sea una mujer dentro del ámbito familiar quien lo haga y en la actualidad ello resulta no solo estigmatizante sino complejo frente a las conquistas -relativas- en el ámbito laboral de las mujeres y la dificultad de dedicarse exclusivamente al cuidado.

La ayuda del Estado a los familiares de las y los usuarios desde la promoción de entornos afectivos y saludables es sin duda otro punto a discutir, dado que no todas las familias se encuentran ensambladas y con las dinámicas y los recursos necesarios para avocarse a la asistencia y el cuidado de las personas con padecimiento mental, sin poder cumplir la expectativa del equipo de salud y ocupando entonces un espacio en los dispositivos públicos mencionados apareciendo el Estado frente a tal falencia de la esfera privada.

\section{Conclusiones}

Con todo lo expuesto, puede interpretarse que el avance en las políticas públicas destinadas a la salud mental comunitaria y al modelo propuesto por la CDPD, la LNSMA y el PNSM, ha tenido grandes avances y cuenta con bases sólidas en su aspecto normativo y doctrinario así como con experiencias formidables en distintos puntos del país.

El desafío es lograr una integralidad en tales políticas, que pasen de ser experiencias aisladas a funcionar como modelo único en salud mental y como instrumento óptimo y eficaz, sin ninguna barrera de las antes mencionadas. Para ello pueden reforzarse las medidas planteadas o pensar en otras formas de apoyo económico y de cambio sociocultural, con su foco en la promoción, garantía y reconocimiento de todos los derechos humanos de las personas usuarias -no solo su derecho la salud -; la realidad es compleja y las políticas sociales no pueden destinarse a cada caso en particular porque perderían su objetivo, si pueden contar con un mayor grado de adaptación.

Lo que no debe perderse de vista es que todos los Estados deben adoptar medidas que impliquen la progresividad en materia de derechos y que las personas, como titulares de los mismos, deben impulsar reformas para que ello no quede varado en una simple recomendación de organismos internacionales; la innegable utilidad del derecho internacional de los 
derechos humanos es su potestad para orientar el desarrollo de las naciones conforme a lo establecido por las convenciones y el marco normativo internacional. Las metas planteadas por el PNSM podría afirmarse que están acorde a ello, sin embargo, se requiere de mayor información, registros y estadísticas para poder evaluarlo desde las políticas públicas especificas (su implementación, gestión, resultado e impacto) tomando a los indicadores en materia de derechos humanos como herramienta compleja y competente para "reflejar los esfuerzos hechos por el estado para satisfacer plenamente los derechos humanos" (Courtis, 2010: 388).

Implementar los indicadores mencionados, así como contar con registros y estadísticas completos y fiables para hacerlo, daría un escenario más claro y permitiría definir con mayor certeza los aciertos y desaciertos a lo largo de la puesta en marcha del PNSM y el camino que es preferible adoptar para continuar; ello también es fundamental en "su potencial utilización por parte de la ciudadanía, tanto como herramienta clave de empoderamiento" (Pautassi, 2010) dado que, ciertamente, "no puede haber un Estado mejor que la ciudadanía que lo sustenta" (Demo y Nunes de Aranha Olivera, 1997: 26) promoviendo las capacidades de los/as ciudadanas/os; principalmente en estos temas, relacionados a la salud mental.

La construcción de una Red en salud mental comunitaria con alternativas adecuadas a las necesidades de cada usuario/a debe contar con el tiempo y la perseverancia como "dos factores que dan sostenimiento a estas formas de abordar la salud mental de personas concretas desde un enfoque de derechos humanos" (Franco, 2015: 261), repudiando las corrientes que consideran que exclusiones como las que ocurren son consecuencia de las realidades individuales y no del sistema -estatal, familiar, económico, neoliberal, entre otros- que cada persona experimenta independientemente de su voluntad.

La posibilidad de "romper" con este círculo vicioso entre vulneración de derechos y problemáticas de salud mental se encuentra dada por la formulación de políticas públicas integrales, interdisciplinarias e intersectoriales, en el marco de la discusión de modelos económicos respetuosos del conjunto de derechos humanos (Yoma, 2018:259).

Además, el desafío de generar políticas integrales no solo en sus dispositivos, sino también en la unión de diversas disciplinas, saberes y recursos, implica también un desafío conforme al vasto territorio argentino al que se hace referencia. Ello requiere de diagnósticos y registros certeros; "dado que el país presenta diversas realidades regionales, es importante contar con información actualizada y oportuna que las refleje" (Sola et al., 2015:20).

Posibilitar espacios de diálogo en la comunidad, con especial hincapié en las voces de los/as usuarios/as, ex usuarios/as y sobrevivientes de la psiquiatría en el debate público, recuperando sus experiencias y escuchando sus necesidades, resulta un punto fundamental. Tal como se viene implementando en organismos y organizaciones internacionales, quitar el discurso patologizante y asistencial de las personas protagonistas no sólo es un deber, sino que también es una posible solución a muchas de las barreras aquí plasmadas en lo que refiere a las miradas del equipo de salud, del grupo familiar o afectivo y de la propia persona. Continuar con las significativas conquistas en salud mental y derechos humanos implica entendernos dentro de un entramado social, donde todos/as cuentan con sus propios padeceres y dificultades, reconociendo que nadie se salva solo/a.

\section{Referencias}

Abramovich, V. y Pautassi, L. (2009). El enfoque de derechos y la institucionalidad de las politicas sociales. Buenos Aires: Editores del Puerto. En línea: enlace.

Aquila, S., Aquino, J., y Bartoli, L. (2015). Un modelo de intervención comunitaria en el campo de la salud mental. la experiencia albariño. En Dirección Nacional de Salud Mental y Adicciones (ed.), Políticas Publicas de Salud Mental. Enfoques, estrategias y experiencias con base en la comunidad. En línea: enlace.

Asociación Civil por la Igualdad y la Justicia (2018). Informe de análisis presupuestario. acciones de promoción de la salud mental comunitaria. En línea: enlace.

Battaglia, M., Brovelli, K., y Castrogiovanni, N. (2019). ¿por qué la familia no se los lleva? políticas públicas, redes sociofamiliares y necesidades de cuidado de las personas con internaciones prolongadas en hospitales públicos de la ciudad de buenos aires. Margen: revista de trabajo social y ciencias sociales, 93. En línea: enlace.

Cea Madrid, J. C. (2019). "Locos por nuestros derechos": Comunidad, salud mental y ciu- 
dadanía en el chile contemporáneo. Quaderns de Psicologia, 21(2):1502. DOI: 10.5565/rev/qpsicologia.1502.

Cohen, J. M. (2015). La casa de ramos mejía. salud mental comunitaria en el marco de las políticas de salud mental de la provincia de buenos aires. Facultad Latinoamericana de Ciencias Sociales, Tesis. En línea: enlace.

Courtis, C. (2010). Apuntes sobre la elaboración y utilización de indicadores en materia de derechos económicos, sociales y culturales. En Arcidiácono, P., Espejo Yaksic, N., y Rodríguez Garavito, G. (eds.), Derechos sociales: justicia, política y economía en América Latina.

D‘Agostino, A. (2016). Políticas sociales en salud mental y transformaciones del estado en argentina (1945-1990). Acta Psiquiátrica y Psicológica de América Latina, 62(2):127-139. En línea: enlace.

De Lellis, M. (2013). Salud mental en la agenda de las políticas públicas de salud. Aletheia, 41: 8-23. En línea: enlace.

Del Valle, D. H. y Boga, D. J. (2016). Desarrollo económico, sistema político y protección social en américa latina. Margen: revista de trabajo social y ciencias sociales, 80 . En línea: enlace.

Demo, P. y Nunes de Aranha Olivera, L. L. (1997). Ciudadania y derechos humanos desde la perspectiva de las políticas públicas. Santiago: Naciones Unidas-CEPAL.

Dirección Nacional de Salud Mental y Adicciones (2013). Plan Nacional de Salud Mental.

Dirección Nacional de Salud Mental y Adicciones (2018a). Dispositivos de inclusión habitacional. En línea: enlace.

Dirección Nacional de Salud Mental y Adicciones (2018b). Abordaje de la salud mental en hospitales generales. En línea: enlace.

Franco, G. (2015). Salud mental y derechos humanos: una experiencia de externación en el territorio. En de Salud Mental y Adicciones, D. N. (ed.), Políticas Publicas de Salud Mental. Enfoques, estrategias y experiencias con base en la comunidad, pp. 253-265. En línea: enlace.
Franco-Giraldo, Á. y Álvarez Dardet, C. (2008). Derechos humanos, una oportunidad para las políticas públicas en salud. Gaceta Sanitaria, 22 (3):280-286. DOI: $10.1157 / 13123975$.

Gerlero, S., Augsburger, A., y Duarte, M. (2011). Salud mental y atención primaria. accesibilidad, integralidad y continuidad del cuidado en centros de salud, argentina. Rev. Argentina en Salud Publica, 2(9):24-29. En línea: enlace.

Levy, E. (2007). Políticas públicas de formación para el trabajo como estrategia de inclusión social. historias de la argentina neoliberal. En $V$ Congreso Latinoamericano de Sociología del Trabajo. En línea: enlace.

Levy, E. y Bermúdez, Á. (2012). De la empleabilidad a la autogestión. la relevancia de la educación formal en el programa "argentina trabaja". En Pautassi, L. y Gamullo, G. (eds.), ¿Mas derechos, menos marginaciones? Politicas sociales y bienestar en la Argentina, pp. 181-221. Buenos Aires: Biblos.

Morasso, A. M. (2018). Gestión del modelo de desinstitucionalización de adultos con alteraciones mentales en el sistema público de la provincia de buenos aires. Universidad Nacional de La Plata, Facultad de Cs. Económicas, Tesis.

Orellana, Y. (2019). Salud mental comunitaria en amba: una reflexión necesaria. repensando las prácticas profesionales desde tres experiencias de abordaje integral de la salud mental. Debate Público, 9(17):85-95. En línea: enlace.

Organización Mundial de la Salud (2005). Conjunto de Guías sobre Servicios y Politicas de Salud Mental. EDIMSA. En línea: enlace.

Pautassi, L. (2010). Indicadores en materia de derechos económicos, sociales y culturales. más allá de la medición. En Abramovich, V. y Pautassi, L. (eds.), La medición de derechos en las politicas sociales. Editores del Puerto. En línea: enlace.

REDI (2011). El derecho a la autonomía de las personas con discapacidad como instrumento para la participación social. Red por los Derechos de las Personas con Discapacidad. En línea: enlace.

Sola, M., Martínez Calle, S., y Cuello, M. (2015). Epidemiologia de la salud mental y las adicciones en argentina: una aproximación general. En 
Dirección Nacional de Salud Mental y Adicciones (ed.), Políticas Publicas de Salud Mental. Enfoques, estrategias y experiencias con base en la comunidad, pp. 19-25. Buenos Aires: Ministerio de Salud de la Nación. En línea: enlace.

Vitelli, A. y Fruttero, E. (2018). Salud mental y derecho derechos sociales e intersectorialidad. En Monasterolo, N. y Rossetti, A. (eds.), Salud Mental y Derecho Derechos Sociales e Intersectorialidad, pp. 113-123. Córdoba: Espartaco. En línea: enlace.

Vázquez, D. y Delaplace, D. (2011). Políticas públicas con perspectiva de derechos humanos: Un campo en construcción. SUR-Revista Internacional de Derechos Humanos, 8(14):35-67. En línea: enlace.

Yoma, S. (2018). Derechos Sociales y Salud Mental: De la vulneración de derechos y la exclusión social a políticas públicas integrales. Córdoba: Espartaco. 